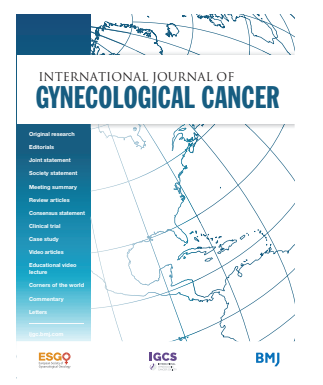

${ }^{1}$ Gynecology Service, Department of Surgery, Memorial Sloan Kettering Cancer Center, New York, New York, USA

2Department of Pathology, Memorial Sloan Kettering Cancer Center, New York, New York, USA

${ }^{3}$ Department of Radiation Oncology, Memorial Sloan Kettering Cancer Center, New York, New York, USA

Correspondence to Dr Mario M. Leitao, Jr., Memorial Sloan Kettering Cancer Center, New York, NY 10065, USA; leitaom@mskcc. org

Received 5 May 2020 Revised 12 June 2020 Accepted 15 June 2020 Published Online First 20 July 2020
D) Check for updates

(C) IGCS and ESGO 2020. No commercial re-use. See rights and permissions. Published by BMJ.

To cite: Pedra Nobre S, Mueller JJ, Gardner GJ, et al. Int J Gynecol Cancer 2020;30:1162-1168.

\title{
Comparison of minimally invasive versus open surgery in the treatment of endometrial carcinosarcoma
}

Silvana Pedra Nobre, ${ }^{1}$ Jennifer J. Mueller, ${ }^{1}$ Ginger J. Gardner, ${ }^{1}$ Kara Long Roche, ${ }^{1}$ Carol L Brown, ${ }^{1}$ Robert A Soslow, ${ }^{2}$ Kaled M. Alektiar, ${ }^{3}$ Yukio Sonoda, ${ }^{1}$ Vance A. Broach, ${ }^{1}$ Elizabeth L. Jewell, ${ }^{1}$ Oliver Zivanovic, ${ }^{1}$ Dennis S Chi, ${ }^{1}$ Nadeem R Abu-Rustum, ${ }^{1}$ Mario M. Leitao, Jr. ${ }^{1}$

\section{HIGHLIGHTS}

- We compared oncologic outcomes between minimally invasive surgery and open surgery in stage I endometrial carcinosarcoma

- Those who underwent minimally invasive surgery had shorter operative time and hospital stays with fewer postoperative complications

- Minimally invasive surgery was not associated with poorer oncologic outcomes

\section{ABSTRACT}

Objective The aim of this study was to compare perioperative and oncologic outcomes between minimally invasive and open surgery in the treatment of endometrial carcinosarcoma.

Methods We retrospectively identified all patients with newly diagnosed endometrial carcinosarcoma who underwent primary surgery via any approach at our institution from January 2009 to January 2018. Patients with known bulky disease identified on preoperative imaging were excluded. The $\chi^{2}$ and Mann-Whitney $\mathrm{U}$ tests were used to compare categorical and continuous variables, respectively. Kaplan-Meier curves were used to estimate survival, and compared using the log rank test. Results We identified 147 eligible patients, of whom $37(25 \%)$ underwent an open approach and 110 (75\%) underwent minimally invasive surgery. Within the minimally invasive group, $92(84 \%)$ of 110 patients underwent a robotic procedure and $14(13 \%)$ underwent a laparoscopic procedure. Four minimally invasive cases $(4 \%)$ were converted to open procedures. Median age, body mass index, operative time, stage, complication grade, and use of adjuvant treatment were clinically and statistically similar between groups. Median length of hospital stay in the open group was 4 days (range 3-21) compared with 1 day (range $0-6$ ) in the minimally invasive group $(p<0.001)$. The rates of any 30 -day complication were $46 \%$ in the open and $8 \%$ in the minimally invasive group $(p<0.001)$. The rates of grade 3 or higher complications were $5.4 \%$ and $1.8 \%$, respectively $(p=0.53)$. Median follow-up for the entire cohort was 30 months (range 0.4-121). Two-year progression-free survival rates were $52.8 \%(\mathrm{SE} \pm 8.4)$ in the open group and $58.5 \%(\mathrm{SE} \pm 5.1)$ in the minimally invasive group $(p=0.7)$. Two-year diseasespecific survival rates were $66.1 \%(\mathrm{SE} \pm 8.0)$ and $81.4 \%$ ( $S E \pm 4.1)$, respectively $(p=0.8)$.

Conclusions In patients with clinical stage I endometrial carcinosarcoma, minimally invasive compared with open surgery was not associated with poor oncologic outcomes, but with a shorter length of hospital stay and a lower rate of overall complications.

\section{INTRODUCTION}

Endometrial cancer is the most common gynecologic malignancy in the United States, with more than 65000 new cases diagnosed annually. ${ }^{1}$ While endometrial carcinosarcomas account for less than $5 \%$ of all endometrial malignancies, they are responsible for $15 \%$ of all endometrial cancer deaths, owing to their aggressive nature. ${ }^{2}$ Although treatment previously focused on the sarcomatous component of these tumors, recent studies have demonstrated that the carcinomatous elements typically drive recurrence and progression. ${ }^{3}$ Moreover, carcinosarcomas arise from epithelial to mesenchymal transformation-the carcinomatous component arising initially and giving way to the sarcomatous element. As such, these cancers are now categorized as high-grade endometrial tumors rather than uterine sarcomas. ${ }^{4}$

Endometrial carcinosarcomas behave distinctly from purely carcinomatous endometrial cancers. Extrauterine disease is present at the time of diagnosis in $60 \%$ of cases, and greater than $50 \%$ of cases will recur despite surgery and adjuvant treatment with chemotherapy or radiation therapy. ${ }^{2}$ The estimated 5-year survival rate for patients with endometrial carcinosarcoma ranges from $33 \%$ to $39 \%$. ${ }^{56}$

Endometrial cancer, including carcinosarcoma, is surgically staged with hysterectomy, bilateral salpingo-0ophorectomy, and lymph node assessment. Laparoscopy has become the standard surgical approach for patients with early-stage uterine carcinoma or sarcoma due to the reassuring results of studies such as Gynecologic Oncology Group LAP2 (Laparoscopy Compared With Laparotomy 
forComprehensive Surgical Staging of Uterine Cancer), which demonstrated no deleterious effect of the minimally invasive approach on perioperative or oncologic outcomes. ${ }^{78}$ Recently, the Laparoscopic Approach to Cervical Cancer (LACC) trial reported worse oncologic outcomes in patients undergoing minimally invasive surgery for cervical cancer. ${ }^{9}$ This has led some to reconsider whether minimally invasive surgery is appropriate for patients with high-grade or higher risk endometrial cancer.

A recent lead article reported an association of worse diseasefree survival in patients with intermediate-risk endometrial cancer who underwent robotic-assisted laparoscopy as opposed to an open approach. ${ }^{10}$ In a systematic review of the literature regarding oncologic outcomes with minimally invasive surgery for high-risk endometrial cancers of various histologies, with only two studies including carcinosarcomas (68 cases), oncologic outcomes for carcinosarcomas were not specifically assessed. ${ }^{11}$ Additionally, the Gynecologic Oncology Group LAP2 study noted that only 87 'sarcomas' were enrolled, but whether any were carcinosarcomas is not specified. Another randomized trial (Laparoscopic Approach to Cancer of the Endometrium (LACE)) only enrolled patients with endometrioid endometrial carcinomas. ${ }^{12}$ Our aim was to compare perioperative and oncologic outcomes between minimally invasive surgery and the open approach, specifically in the treatment of clinical stage I uterine carcinosarcoma.

\section{METHODS}

After obtaining approval from our institutional review board, we retrospectively identified all patients with newly diagnosed uterine carcinosarcoma treated at our institution between January 2009 and January 2018. These patients had undergone surgical staging, which included hysterectomy, bilateral salpingo-oophorectomy, and lymph node evaluation via either open (abdominal) surgery or minimally invasive surgery (robotic-assisted or laparoscopy). Patients included in the open procedure group were those planned for laparotomy at the time of diagnosis and surgical consent. The planned minimally invasive surgery group included patients who had a planned and completed minimally invasive surgery, as well as those who had a planned minimally invasive surgery but were converted to laparotomy. Patients with known bulky or extrauterine disease (lymph nodes $\geq 2 \mathrm{~cm}$ in size or disseminated carcinomatosis) identified on standard preoperative imaging with computed tomography or who had received neoadjuvant chemotherapy were excluded from this study.

Relevant patient demographics, as well as medical and surgical history, were abstracted from the medical record. This information included age, body mass index, surgical procedure, surgical stage, tumor characteristics (depth of invasion, lymphovascular invasion), operative time, postoperative complications, length of hospital stay, readmission rates, receipt of adjuvant treatment, and clinical outcomes. All minimally invasive cases were performed with the use of a transcervical uterine manipulator. Intraoperative and postoperative complications are prospectively captured and documented at our institution by dedicated research staff using our published Memorial Sloan Kettering Cancer Center Surgical Secondary Events Grading System. ${ }^{13}$ Surgical staging was performed according to the
International Federation of Gynecology and Obstetrics 2009 staging classification system.

Patterns of recurrence were determined by site of first documented recurrence seen on imaging or physical exam. Progressionfree survival was calculated from the date of surgery to the date of first disease progression, or the date of last known follow-up or death for those who did not recur. Disease-specific survival was calculated from the date of surgery to the date of last known follow-up or confirmed death from disease. Overall survival was calculated from the date of surgery to the date of last known follow-up or death. Descriptive statistics were applied for clinicodemographic data. The $\chi^{2}$ and Mann-Whitney $\mathrm{U}$ tests were used to compare categorical and continuous variables, respectively. Kaplan-Meier curves were used to estimate survival, and compared using log rank statistics.

In accordance with the journal's guidelines, we will provide our data for the reproducibility of this study to other centers, if requested.

\section{RESULTS}

We identified 184 patients with newly diagnosed endometrial carcinosarcoma treated with primary surgery at our institution. Thirtyfive patients were found to have bulky or extrauterine disease on preoperative imaging and two patients had received neoadjuvant chemotherapy; these patients were excluded from analysis. A total of 147 patients met our study inclusion criteria and were analyzed. Patients were planned for an open procedure for presumed earlystage carcinosarcoma if they had an enlarged uterus with narrow vagina, had a multi-lobulated or enlarged fibroid uterus (range 10-20 cm), had undergone combination surgery with other specialties, or had multiple prior abdominal surgeries. None of the patients underwent a planned open procedure due to concerns over the presence of metastatic disease. Patient characteristics as well as surgical approach and perioperative characteristics are shown in Table 1.

The median age for the entire cohort was 67 years (range 50-96). The median age for the open group was 63 years (range 50-86) and the median age for the planned minimally invasive surgery group was 67 years (range $50-96)(p=0.1)$. The median body mass index for the entire cohort was $29.8 \mathrm{~kg} / \mathrm{m}^{2}$ (range 18.9-54.8). The median body mass index for the open group was $28.9 \mathrm{~kg} / \mathrm{m}^{2}$ (range 19-46.8) and the median body mass index for the minimally invasive surgery group was $30.2 \mathrm{~kg} / \mathrm{m}^{2}$ (range $\left.18.9-54.8\right)(p=0.4)$. The breakdown of disease by stage was comparable between the two groups (Table 1). There was no difference in use of adjuvant therapy or type of adjuvant treatment received between the two groups $(p=0.5$ and $p=0.6$, respectively) (Table 1). Heterologous elements were described in 23 patients $(62 \%)$ in the open group and in $42(38 \%)$ in the minimally invasive group $(p=0.02)$. In 10 cases, this determination could not be made or was not specified in the pathology reports. Progression-free, disease-specific, and overall survival rates were not different based on heterologous versus homologous elements for the combined cohort $(p=0.81,0.77$, and 0.96 , respectively).

Thirty-seven patients (25\%) underwent an open approach and $110(75 \%)$ underwent a planned minimally invasive surgery. In the planned minimally invasive surgery group, $92(84 \%)$ underwent 


\section{Original research}

\begin{tabular}{|c|c|c|c|c|}
\hline Variable & Total cohort & Open & Planned MIS & $P$ value \\
\hline Total No of patients & 147 & 37 & 110 & \\
\hline Age (years) (median (range)) & $67(50-96)$ & $63(50-86)$ & $67(50-96)$ & 0.1 \\
\hline Body mass index $\left(\mathrm{kg} / \mathrm{m}^{2}\right)$ (median (range)) & $29.8(18.9-54.8)$ & $28.9(19-46.8)$ & $30.2(18.9-54.8)$ & 0.4 \\
\hline LOS (days) (median (range)) & $1(0-21)$ & $4(3-21)$ & $1(0-6)$ & $<0.001$ \\
\hline Operative time (min) (median (range)) & 187 (83-392) & $156(83-330)$ & $191.5(99-392)$ & 0.04 \\
\hline \multicolumn{5}{|l|}{ Procedure (n (\%)) } \\
\hline Robotic & $92(63)$ & $0(0)$ & $92(84)$ & \\
\hline Laparoscopic & $14(9)$ & $0(0)$ & $14(13)$ & \\
\hline Open & $41(28)$ & $37(100)$ & $4(4)$ & \\
\hline Planned MIS & $110(75)$ & $0(0)$ & $108(100)$ & \\
\hline Completed MIS & $106(96)$ & $0(0)$ & $106(97)$ & \\
\hline Converted & $4(2.7)$ & $0(0)$ & $4(3.6)$ & \\
\hline \multicolumn{5}{|l|}{ Stage $(\mathrm{n}(\%))$} \\
\hline 1 & $92(63)$ & $21(57)$ & $71(65)$ & 0.11 \\
\hline II & $3(2)$ & $0(0)$ & $3(3)$ & \\
\hline III & $32(22)$ & $7(19)$ & $25(23)$ & \\
\hline IV & $20(14)$ & $9(24)$ & $11(10)$ & \\
\hline \multicolumn{5}{|l|}{ Histology (n (\%)) } \\
\hline Homologous & $72(49)$ & $11(30)$ & $61(56)$ & 0.02 \\
\hline Heterologous & $65(44)$ & $23(62)$ & $42(38)$ & \\
\hline Not reported & $10(7)$ & $3(8)$ & $7(6)$ & \\
\hline \multicolumn{5}{|l|}{ Nodal assessment method (n (\%)) } \\
\hline SLN alone & $45(31)$ & $5(14)$ & $40(36)$ & $<0.001$ \\
\hline LND alone & $30(20)$ & $16(43)$ & $14(13)$ & \\
\hline SLN+LND & $61(42)$ & $12(32)$ & $49(45)$ & \\
\hline No assessment & $11(7)$ & $4(11)$ & $7(6)$ & \\
\hline \multicolumn{5}{|l|}{ Nodal basins assessed (n (\%)) } \\
\hline Pelvic only & $94(64)$ & $23(62)$ & $71(65)$ & 0.93 \\
\hline Pelvic and para-aortic & $42(29)$ & $10(27)$ & $32(29)$ & \\
\hline No assessment & $11(7)$ & $4(11)$ & $7(6)$ & \\
\hline Lymph nodes removed (n) (median (range)) & $6(1-45)$ & $6(1-45)$ & $6(1-41)$ & 0.28 \\
\hline \multicolumn{5}{|l|}{ Year of surgery $(\mathrm{n}(\%))$} \\
\hline 2009 & $20(14)$ & $6(16)$ & $14(13)$ & 0.06 \\
\hline 2010 & $6(4)$ & $3(8)$ & $3(3)$ & \\
\hline 2011 & $9(6)$ & $4(11)$ & $5(5)$ & \\
\hline 2012 & $18(12)$ & $3(8)$ & $15(14)$ & \\
\hline 2013 & $18(12)$ & $3(8)$ & $15(14)$ & \\
\hline 2014 & $16(11)$ & $5(14)$ & $11(10)$ & \\
\hline 2015 & $18(12)$ & $3(8)$ & $15(14)$ & \\
\hline 2016 & $21(14)$ & $1(3)$ & $20(18)$ & \\
\hline 2017 & $20(14)$ & $8(22)$ & $12(11)$ & \\
\hline 2018 & $1(<1)$ & $1(3)$ & $0(0)$ & \\
\hline \multicolumn{5}{|l|}{ Adjuvant treatment (n (\%)) } \\
\hline No & $12(8)$ & $4(11)$ & $8(7)$ & 0.5 \\
\hline Yes & 135 (92) & $33(89)$ & $102(93)$ & \\
\hline
\end{tabular}


Table 1 Continued

\begin{tabular}{|c|c|c|c|c|}
\hline Variable & Total cohort & Open & Planned MIS & $P$ value \\
\hline \multicolumn{5}{|c|}{ Type of adjuvant treatment (n (\%)) } \\
\hline None & $12(8)$ & $4(11)$ & $8(7)$ & 0.6 \\
\hline Chemotherapy alone & $34(23)$ & $11(30)$ & $23(21)$ & \\
\hline IVRT alone & $5(3)$ & $1(3)$ & $4(4)$ & \\
\hline WPRT alone & $3(2)$ & $0(0)$ & $3(3)$ & \\
\hline Chemotherapy+IVRT & $85(58)$ & $18(49)$ & $67(61)$ & \\
\hline Chemotherapy+WPRT & $6(4)$ & $2(5)$ & $4(4)$ & \\
\hline Chemotherapy+IVRT/EBRT & $2(1)$ & $1(3)$ & $1(1)$ & \\
\hline
\end{tabular}

EBRT, external beam radiation therapy; IVRT, intravaginal radiation therapy; LND, lymphadenectomy; LOS, length of hospital stay; MIS, minimally invasive surgery; SLN, sentinel lymph node; WPRT, whole pelvic radiation therapy.

a robotic-assisted laparoscopic procedure and $14(13 \%)$ underwent conventional laparoscopy. In the planned minimally invasive surgery group, four patients (4\%) were converted to laparotomy. Three of these patients were converted to laparotomy from a planned robotic-assisted approach and one from a planned conventional laparoscopy. All conversions were due to significant adhesive disease in the abdomen.

Median operative time for the entire cohort was 187 min (range 83-392). Median operative times were $156 \mathrm{~min}$ (range 83-330) for the open group and $191.5 \mathrm{~min}$ (range 99-392) for the planned minimally invasive surgery group $(p=0.04)$. Median length of hospital stay for the entire cohort was 1 day (range 0-21). Median length of stay was 4 days (range 3-21) for the open group and 1 day (range $0-6$ days) for the minimally invasive surgery group $(p<0.001)$.

Twenty-six patients (18\%) experienced at least one postoperative complication of any grade within 30 days of surgery (Table 2). Seventeen patients (46\%) experienced a postoperative complication after an open procedure, and nine patients (8\%) experienced

\begin{tabular}{lcccc}
\hline Table 2 & \multicolumn{1}{l}{ Complications and readmissions } & \\
\hline Variable & Total cohort & Open & Planned MIS & P value \\
\hline Total & 147 & 37 & 110 & \\
30 day complications $(\mathrm{n}(\%))$ & & & \\
No & $121(82)$ & $20(54)$ & $101(92)$ & $<0.001$ \\
Yes & $26(18)$ & $17(46)$ & $9(8)$ & \\
Complication grade (n (\%)) & & & \\
1 & $11(42)$ & $6(35)$ & $5(56)$ & 0.24 \\
2 & $11(42)$ & $9(53)$ & $2(22)$ & \\
3 & $3(12)$ & $2(12)$ & $1(11)$ & \\
4 & $0(0)$ & $0(0)$ & $0(0)$ & \\
5 & $1(4)$ & $0(0)$ & $1(11)$ & \\
Grade $\geq 3$ & complications (n (\%)) & & \\
No & $22(85)$ & $15(88)$ & $7(78)$ & \\
Yes & $4(15)$ & $2(12)$ & $2(22)$ & \\
Readmission & $<30$ days (n (\%)) & & \\
No & $142(97)$ & $34(92)$ & $108(98)$ & \\
Yes & $5(3)$ & $3(8)$ & $2(2)$ & \\
\hline
\end{tabular}

MIS, minimally invasive surgery. a postoperative complication after a planned minimally invasive surgery $(p<0.001)$. The majority of complications were grade 1 or 2 in both the open and planned minimally invasive surgery groups ( $88 \%$ vs $78 \%$, respectively; $p=0.2$ ). There were four grade 3 or higher complications in the entire cohort: $2(5 \%)$ in the 37 patients in the open group and $2(2 \%)$ in the 110 patients in the planned minimally invasive surgery group ( $p=0.2$ for the 26 complications; $\mathrm{p}=0.5$ for all cases).

Five $(3 \%)$ of 147 patients were readmitted within 30 days of surgery: $3(8 \%)$ of the 37 patients in the open group and $2(2 \%)$ of the 110 in the planned minimally invasive surgery group $(p=0.06)$. In the minimally invasive surgery group, one patient was readmitted with a wound infection and separation after conversion to laparotomy, and another was readmitted with decreased range of motion in the left lower extremity, as well as fever. In the open group, one patient was readmitted with ascites and carcinomatosis, one patient for pelvic collection and thigh pain, and one for diarrhea and dehydration.

The median follow-up for the entire cohort was 30 months (range $0.4-121)$. The median follow-up for the open group was 24.6 months (range 1-110), and the median follow-up for the minimally invasive surgery group was 30.8 months (range $0.4-121)(p=0.88)$. There were 71 recurrences $(48 \%)$ in the entire cohort: $19(51 \%)$ among the 37 patients in the open group and $52(47 \%)$ among the 110 in the planned minimally invasive surgery group $(p=0.6)$. There was no difference in patterns of recurrence between the two groups. Most recurrences were extra-pelvic peritoneal (43\% vs $29 \%$ in the open and planned minimally invasive surgery groups, respectively; $p=0.9$ ) (Table 3 ).

There were no differences in progression-free survival, diseasespecific survival, or overall survival between the two groups. The 2-year progression-free survival rates were $52.8 \%$ in the open group and $58.5 \%$ in the minimally invasive surgery group $(\mathrm{p}=0.7)$ (Figure $1 \mathrm{~A})$. The 2-year disease-specific survival rates were $66.1 \%$ and $81.4 \%$, respectively ( $p=0.7$ ) (Figure 1B). The 2-year overall survival rates were $64.3 \%$ and $74.6 \%$, respectively ( $p=0.8$ ) (Figure $1 C$ ). We noted that the survival curves for progression-free, disease-specific, and overall survival crossed at approximately the $40-48$ month time point. This may reflect a time dependence of the outcome between groups. We tested whether the Cox proportional hazards assumption had been violated, but the log minus log plots were symmetrical without crossing throughout all time points. Furthermore, the Cox regression analysis 


\section{Original research}

\begin{tabular}{lllll}
\hline Table 3 Patterns of recurrence & & & & \\
\hline & Total cohort & Open & Planned MIS & P value \\
\hline $\mathrm{N}$ & 71 & 19 & 52 & \\
Time to recurrence (months) (median (range)) & $12.4(1-59)$ & $8.45(1-35)$ & $16.02(1-59)$ & 0.03 \\
Pattern of recurrence ( $(\%))$ & & & & 0.9 \\
Pelvic non-vaginal & 11 & $3(16)$ & $8(15)$ & \\
Vaginal & 4 & $1(5)$ & $3(6)$ & \\
Peritoneum & 24 & $8(42)$ & $16(29)$ & \\
Hematogenous $\dagger$ & 9 & $2(10)$ & $6(13)$ & \\
Nodal & 8 & $2(10)$ & $11(21)$ & \\
Multi-site & 14 & $3(16$ & $1(2)$ \\
$\quad$ Otherf & 1 & $0(0)$ & \\
\hline
\end{tabular}

*Peritoneum, outside the pelvis.

†Liver, lung, bone, and brain.

‡Vulvar.

MIS, minimally invasive surgery

with time-dependent covariate was not significant for progressionfree, disease-specific, and overall survival. The HRs adjusted for the presence of heterologous elements were 1.10 (95\% Cl 0.64 to 1.91; $\mathrm{p}=0.75), 1.10$ (95\% Cl 0.58 to 2.09; $\mathrm{p}=0.77)$, and 0.93 (95\% Cl 0.51 to $1.71 ; p=0.96)$, respectively.

In patients with surgical stage I disease alone, there were no differences in progression-free, disease-specific, or overall survival between the open and planned minimally invasive surgery groups. The 2-year progression-free survival rates were $65.9 \%$ in the open group and $64 \%$ in the minimally invasive surgery group $(p=0.8)$. The 2-year disease-specific survival rates were $75 \%$ and $83.5 \%$, respectively $(\mathrm{p}=0.5)$. The 2 -year overall survival rates were $71.4 \%$ and $78.9 \%$, respectively $(\mathrm{p}=0.5)$.

\section{DISCUSSION}

In this cohort of patients with clinical stage I endometrial carcinosarcoma treated at our center, minimally invasive surgery was
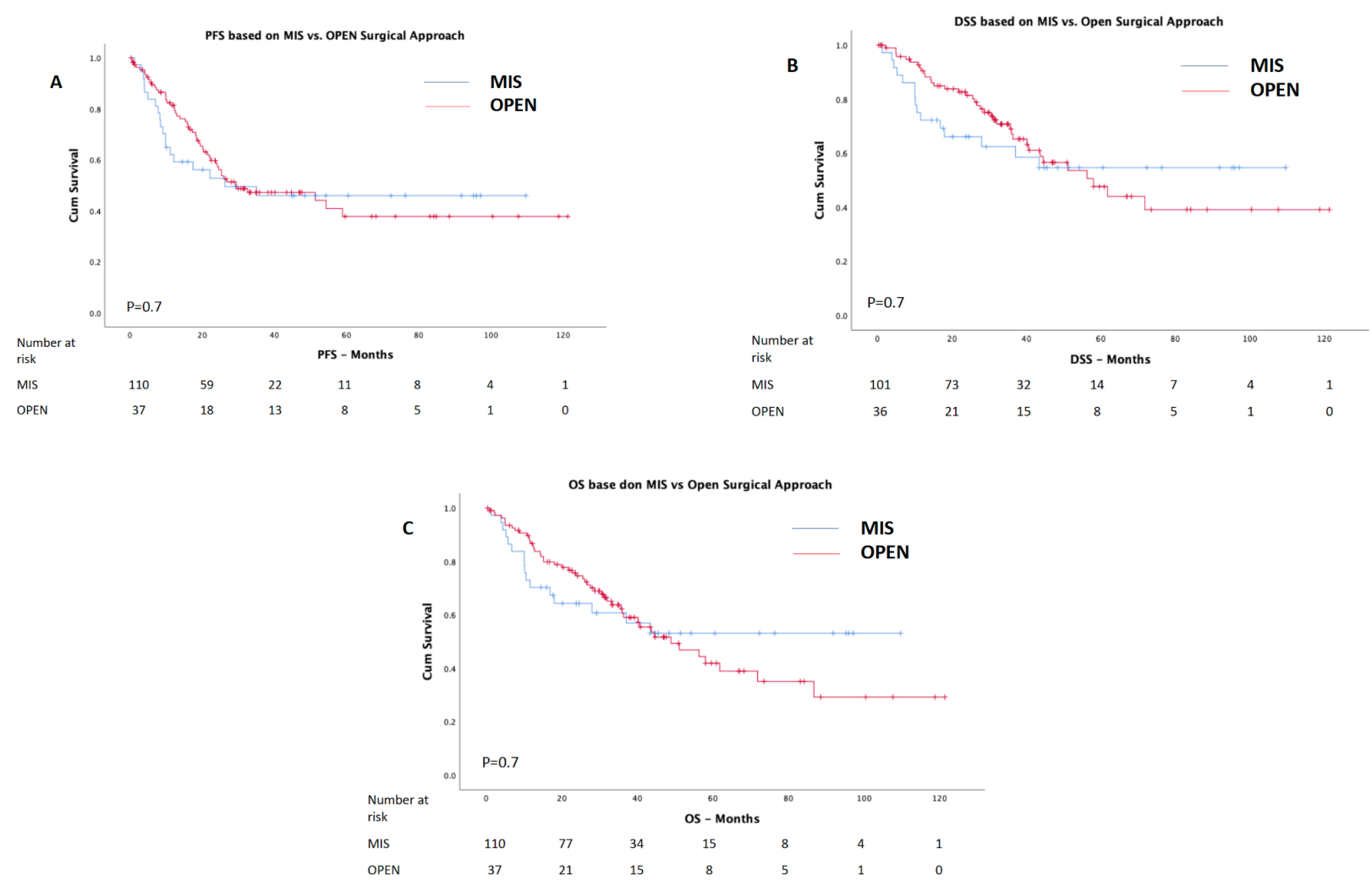

Figure 1 (A) Progression-free survival, minimally invasive surgery (MIS) versus open surgery. (B) Disease-specific survival, MIS versus open surgery. (C) Overall survival, MIS versus open surgery. DSS, disease-specific survival; OS, overall survival; PFS, progression-free survival. 
associated with a shorter length of hospital stay, shorter operative time, and fewer perioperative complications. Progression-free and overall survival did not differ between patients who underwent minimally invasive surgery versus open surgery.

The benefits of minimally invasive surgery are well documented in the gynecologic oncology literature. ${ }^{71^{114-16}}$ Bergstrom et al evaluated the rates of minimally invasive surgery for endometrial cancer at high-volume National Comprehensive Cancer Network centers and concluded that disparities in age, race, and body mass index seen in studies based on national databases were not observed at these centers, and the rates of minimally invasive surgery at these centers were higher than the national average, all while having low perioperative complications. ${ }^{14}$ In the landmark LAP2 study, Walker et al demonstrated the safety and feasibility of minimally invasive surgery in endometrial cancer, without compromise to oncologic outcomes in these patients. ${ }^{78}$ These patients had less postoperative pain, shorter recovery periods, and shorter length of hospital stay, without an increase in rates of complications, intraoperative injuries, or compromise to survival. ${ }^{7}$ Although eligible for LAP2, patients with uterine carcinosarcoma represented only $1.6 \%$ of the study cohort, if we are to assume that they were the 'sarcomas' in the cohort. Due to the small number of patients in this subgroup, the authors could not comment specifically on minimally invasive surgery in carcinosarcoma and its influence on perioperative or oncologic outcomes in this highgrade histology. ${ }^{28}$ The LACE trial did not include carcinosarcomas. ${ }^{12}$

There have been no prospective studies evaluating surgical route in patients with high-risk endometrial cancer alone. Randomized studies in this group are particularly challenging due to the disease's rarity. Recently, Scaletta et al performed a systematic review of the literature evaluating surgical approach in highrisk endometrial cancer. ${ }^{11}$ The review included a total of nine studies published between 2012 and 2019, and grouped patients according to high-risk endometrial subtype, including papillary serous, carcinosarcoma, clear cell, and grade 3 endometrioid carcinomas. Scaletta et al concluded that minimally invasive surgery is a safe platform for the management of patients with high-risk endometrial cancer, with improved perioperative and postoperative outcomes and comparable oncologic outcomes to those of open surgery. Only one of the studies included in the review, however, included carcinosarcomas. Koskas et a ${ }^{15}$ investigated the oncologic outcomes of patients undergoing minimally invasive surgery versus an open procedure for endometrial cancer at three institutions. Fifteen percent of the cohort had carcinosarcoma, and on univariate and multivariate analyses, histology (specifically carcinosarcoma) was found to be associated with worse disease-free and overall survival. However, the authors found no difference in 5-year disease-specific or overall survival between the minimally invasive surgery and open groups.

Since the provocative results of the LACC trial ${ }^{9}$ regarding oncologic outcomes with minimally invasive surgery in patients with early-stage cervical cancer, there has been a new debate focused on the role of minimally invasive surgery in high-risk endometrial cancer. While Scaletta et a ${ }^{11}$ provided a comprehensive overview of the available literature, with results similar to those of the LAP2 trial, there was significant under-representation of carcinosarcoma in most of the study cohorts included in the review. Our study provides a 10-year composite experience demonstrating reduced perioperative morbidity and reassuring oncologic outcomes when minimally invasive surgery is used for clinical stage I endometrial carcinosarcoma.

Lymphadenectomy was performed more often in the open group in our cohort. However, the nodal basins assessed were similar between the groups. Our recent analysis assessing outcome in carcinosarcoma comparing sentinel lymph node mapping to lymphadenectomy noted no association with oncologic outcome and, therefore, we did not feel an adjustment was merited. ${ }^{17}$ The higher rate of lymphadenectomy, however, may have contributed somewhat to the postoperative complication differences. There was also concern that there may have been time dependence in progression-free, disease-specific, and overall survival. We tested for this and found no association. However, the median follow-ups were shorter than the crossing point, and there were even fewer at-risk cases after that time point. While statistically not significant, it may be that something is happening between groups at $40-48$ months. It may also be a factor of the differing follow-up times and smaller numbers. We plan to continue close follow-up of these cohorts and hope to provide updated outcomes in the future. This is also the reason we chose to report 2-year outcomes.

The limitations of our analysis include the inherent bias of retrospective studies and that patients were from a single institution. To strengthen our analysis and minimize cohort heterogeneity, we included only clinical stage I carcinosarcoma with negative preoperative imaging and pathology reviewed by expert gynecologic pathologists. We could not require pathologic confirmation of recurrence, as this was a retrospective study analyzing measurements of outcomes that were obtained clinically. Pathologic confirmation is not routinely clinically considered or required in cases of obvious clinical and radiologic recurrence or progression, especially in malignancies with a known high recurrence risk. This is in line with the majority of other retrospective analyses. We do not feel this limits the interpretation of our findings, beyond that the study was retrospective, but some may. Another limitation is that the open and minimally invasive groups may not have been truly the same, as the patients in the open cohort were deemed unsuitable for minimally invasive surgery by the surgeon. However, the reasons were not directly related to tumor features, and cases with any extrauterine disease preoperatively were not included in this analysis. The strengths of our study include patients treated at a high-volume comprehensive cancer center by expert gynecologic oncologists.

In conclusion, women with clinical stage I carcinosarcoma treated with minimally invasive surgery compared with laparotomy experienced shorter length of hospital stay, shorter operative time, and fewer postoperative complications. Recurrence rates and survival outcomes were similar between the two cohorts, suggesting that minimally invasive platforms can be considered for patients diagnosed with clinical stage I carcinosarcoma. A randomized trial would be needed to confirm our results, as with any other retrospective hypothesis-generating study. However, this would need to be a non-inferiority study, requiring an even larger number of patients than a superiority study. Considering the rarity of this tumor, it seems unlikely that such a randomized non-inferiority trial will ever be feasible.

Twitter Vance A. Broach @VanceBroach and Mario M. Leitao, Jr. @leitaomd 


\section{Original research}

Contributors Conceptualization, formal analysis, investigation, methodology, and roles/writing: SPN and ML. Data curation: SPN. Funding acquisition, project administration, supervision, and validation: ML. Writing — review and editing: all authors. Final approval: all authors.

Funding This research was funded in part through the National Institutes of Health/National Cancer Institute Cancer Center Support Grant P30 CA008748.

Competing interests ML is an ad hoc speaker for Intuitive Surgical, Inc. Outside the submitted work, ML reports personal fees from JNJ/Ethicon. EJ reports personal fees from Covidien/Medtronic. DSC reports personal fees from Bovie Medical Co, Verthermia Inc (now Apyx Medical Corp), C Surgeries, and Biom 'Up. NRA-R reports grants from Stryker/Novadaq, Olympus, and GRAlL.

Patient consent for publication Not required.

Ethics approval The study was approved by our institutional review board.

Provenance and peer review Not commissioned; externally peer reviewed.

Data availability statement Data are available upon reasonable request. For data please contact the corresponding author.

\section{REFERENCES}

1 Siegel RL, Miller KD, Jemal A, et al. Cancer statistics, 2020. CA A Cancer J Clin 2020;70:7-30.

2 Cantrell LA, Blank SV, Duska LR. Uterine carcinosarcoma: a review of the literature. Gynecol Oncol 2015;137:581-8.

3 McCluggage WG. Uterine carcinosarcomas (malignant mixed Mullerian tumors) are metaplastic carcinomas. Int J Gynecol Cancer 2002;12:687-90.

4 Kurman RJ, Carcangiu ML, Herrington CS, et al, eds. WHO classification of tumours of female reproductive organs. 4th edn. Geneva: World Health Organization, 2014.

5 Schweizer W, Demopoulos R, Beller U, et al. Prognostic factors for malignant mixed Müllerian tumors of the uterus. Int J Gynecol Pathol 1990;9:129-36.

6 Wolfson AH, Brady MF, Rocereto T, et al. A Gynecologic Oncology Group randomized phase III trial of whole abdominal irradiation (WAI) vs. cisplatin-ifosfamide and mesna (CIM) as post-surgical therapy in stage I-IV carcinosarcoma (CS) of the uterus. Gynecol Oncol 2007; 107:177-85.

7 Walker JL, Piedmonte MR, Spirtos NM, et al. Laparoscopy compared with laparotomy for comprehensive surgical staging of uterine cancer: Gynecologic Oncology Group Study LAP2. J Clin Oncol 2009;27:5331-6.

8 Walker JL, Piedmonte MR, Spirtos NM, et al. Recurrence and survival after random assignment to laparoscopy versus laparotomy for comprehensive surgical staging of uterine cancer: Gynecologic Oncology Group LAP2 Study. J Clin Oncol 2012;30:695-700.

9 Ramirez PT, Frumovitz M, Pareja R, et al. Minimally invasive versus abdominal radical hysterectomy for cervical cancer. N Engl J Med 2018;379:1895-904.

10 Song J, Le T, Hopkins L, et al. A comparison of disease recurrence between robotic versus laparotomy approach in patients with intermediate-risk endometrial cancer. Int J Gynecol Cancer 2020;30:160-6.

11 Scaletta G, Dinoi G, Capozzi V, et al. Comparison of minimally invasive surgery with laparotomic approach in the treatment of high risk endometrial cancer: a systematic review. Eur J Surg Oncol 2020;46:782-8.

12 Janda M, Gebski V, Davies LC, et al. Effect of total laparoscopic hysterectomy vs total abdominal hysterectomy on diseasefree survival among women with stage I endometrial cancer: a randomized clinical trial. JAMA 2017;317:1224-33.

13 Strong VE, Selby LV, Sovel M, et al. Development and assessment of Memorial Sloan Kettering cancer center's surgical secondary events grading system. Ann Surg Oncol 2015;22:1061-7.

14 Bergstrom J, Aloisi A, Armbruster S, et al. Minimally invasive hysterectomy surgery rates for endometrial cancer performed at National Comprehensive Cancer Network (NCCN) centers. Gynecol Oncol 2018;148:480-4.

15 Koskas M, Jozwiak M, Fournier M, et al. Long-term oncological safety of minimally invasive surgery in high-risk endometrial cancer. Eur J Cancer 2016;65:185-91.

16 Fader AN, Seamon LG, Escobar PF, et al. Minimally invasive surgery versus laparotomy in women with high grade endometrial cancer: a multi-site study performed at high volume cancer centers. Gynecol Oncol 2012;126:180-5.

17 Schiavone MB, Zivanovic O, Zhou Q, et al. Survival of patients with uterine carcinosarcoma undergoing sentinel lymph node mapping. Ann Surg Oncol 2016;23:196-202. 